\title{
Analysis of Factors Affecting Output Levels and Frequencies of MP3 Players
}

\author{
Jinsook Kim \\ Division of Speech Pathology and Audiology, College of Natural Sciences, Hallym University, Chuncheon, Korea
}

$\begin{array}{ll}\text { Received } & \text { July 29, 2013 } \\ \text { Revised } & \text { August 1, 2013 } \\ \text { Accepted } & \text { August 5, 2013 }\end{array}$

Address for correspondence Jinsook Kim, PhD

Division of Speech Pathology and Audiology,

College of Natural Sciences,

Hallym University,

1 Hallimdaehak-gil,

Chuncheon 200-702, Korea

Tel +82-33-248-2213

Fax +82-33-256-3420

E-mail jskim@hallym.ac.kr
Exposure to high levels of music that could lead to music induced hearing loss (MIHL) has been of recent interest especially for young adults, considering their excessive use of personal listening devices such as MP3 player. More attention should be drawn to MIHL for noting that early noise exposure leads to earlier onset of presbycusis. In search of appropriate and safe listening habits for young adults, this investigation was aimed to evaluate output levels and frequencies generated by the Samsung galaxy note MP3 player depending on two earphone types; earbud and over-the-ear earphones and three music genres; rock, hip-hop, ballade. A sound level meter was used to measure output level and frequency spectrum between 12.5 and $16000 \mathrm{~Hz}$ at all 1/3-octave bands. The following results can be summarized. 1) The earphone styles did not produce significant difference in output levels, but the music genres did. However, the results of music genres varied. 2) Neither earphone styles nor music genres produced significant difference in frequency response spectrum, except music genres at the volume settings we usually listen to. Additionally, volume levels should be lower than $50 \%$ for usual listening situation. Through this investigation, it was noted that the frequency range was substantial between 50 and $1000 \mathrm{~Hz}$ regardless of the styles of earphones and music genres, implying that we should be cautious of this frequency range when we listen to music. Researchers should give more attention to the effects of the mixture of output level and frequency spectrum, considering that the auditory system has frequency specificity from the periphery to the central to provide refined methods for protecting our ears from MIHL.

Korean J Audiol 2013;17:59-64 spectrum.

\section{Introduction}

Excessive noise exposure impairs the ability to communicate and, in high enough doses, inflicts permanent damage to the auditory system, which is called noise induced hearing loss (NIHL). ${ }^{1)}$ NIHL is the second most common form of acquired hearing loss following age-related hearing loss. ${ }^{2)}$ Other than occupational and military noise exposure, exposure to high levels of music that could lead to NIHL has been of recent interest in literatures. Notably, the term music induced hearing loss (MIHL) was used in the literature. ${ }^{3-5)}$ MIHL refers to NIHL by listening to loud output levels of music for long periods of time. Possible causes of MIHL are urban music clubs, concert halls, discotheques, and personal listening devices (PLDs).

Of these, there are growing concerns over noise exposure via PLDs used by young adults. The capacity for PLDs' output levels presenting the risk of MIHL has been reported by many researchers. ${ }^{6,7)}$ Although some argue that listening to music does not damage hearing, sufficient evidence has confirmed that the risk is real. It has been shown that there is a significant correlation between PLD listening behavior and auditory function. ${ }^{8)}$ Considering the fact that early noise exposure leads to earlier onset of presbycusis, ${ }^{9)}$ it is clear that the hearing health of young adults living in this industrial and noisy society is in jeopardy. Fortunately, a survey reported that many young people are aware of this jeopardy. ${ }^{10)}$ Yet, awareness of danger does not necessarily mean knowledge to avoid the danger.

\section{Factors That Could Affect Output Levels of PLDs}

Listening output levels of PLDs are known to vary depend- 
ing on the background noise, music genres, and earphone styles. Generally, people seek a signal-to-noise ratio of $+13 \mathrm{~dB}$ for comfortable listening environments. ${ }^{11)}$ However, ambient background noise cannot be easily controlled. Consequently, listeners usually increase the volume control to overcome masking of music from noise. For example, on a busy city street in urban Australia, an investigation reported that listening level exceeded 100 dBA free-field equivalents in passers-by. ${ }^{6}$ Another research on the relation between background noise levels and the listening levels revealed that the comfortable average music levels were increased by 7 to $13 \mathrm{~dB}$, in quiet and noisy background, respectively. ${ }^{12)}$

Assuming spectral and temporal pattern will be different according to music genre, the output levels and frequency characteristics of sound will also change depending on music genres. Fligor and $\mathrm{Cox}^{4)}$ measured output levels of eight different music genres relative to white noise levels at maximum volume setting in the research for determining output levels signal used. The results showed that rock, country, and adult contemporary music samples were very similar to white noise levels, but samples of rap/R\&B, pop, classical, dance, and jazz were overestimated by $2.5,3.9,4.9,5.6$, and $12 \mathrm{~dB}$, respectively. Results also demonstrated that the highest peak in the sample and the number of peaks in 10-seconds were different according to music genres for different combinations of CD players and headphones. However, it was reported that similar output levels among samples of rock, $R \& B$, country, dance, and top forty music at maximum volume settings. ${ }^{13)}$ Further, in the research on short-term auditory effects after listening to 17 compiled pop-rock songs for 1 hour, Keppler, et al. ${ }^{14)}$ found out a distinct peak in the spectrum of over-the-ear earphones at about $2.5 \mathrm{kHz}$. Accordingly, between 2.0 and $2.8 \mathrm{kHz}$, hearing threshold changes were noted with the transient-evoked otoacoustic emission amplitude measurements after exposure. To verify that music genre is one of the factors that produce considerable variability, the frequency data of each music should be further investigated.

Many investigations reported that generally, smaller headphones had higher output levels. Although method and materials varied, many researchers reported similar results that ear-bud earphones could increase 7-9 dB, $5.5 \mathrm{~dB}$, and $5.2 \mathrm{~dB}$ in output levels in comparison to those of over-the-ear earphones. ${ }^{4,13)}$ The degree of sound isolation provided by overthe-ear earphones in noisy listening environments presumably allowed subjects to choose lower volume levels. Further, preferred listening levels of the two different earphones, over-theear and ear-bud earphones in quiet and noisy background environments were explored. And it was concluded that the covering of ears by over-the-ear earphones provided passive sound isolation as well as active noise cancellation. ${ }^{11)}$

Although advanced researches about PLDs and earphones were basically focused on preferred listening levels of subjects, output levels and frequency ranges according to types of earphones and music genres should also be considered for a more accurate cause and effect relationship verification. Aware of this, finding for appropriate and safe listening habits for young adults, the output levels and frequencies generated depending on two earphone types and three music genres were measured in decibels at medium and maximum volume settings by a sound level meter.

\section{Materials and Experimental Procedures}

Twenty earphones, ten over-the-ear and ten ear-bud earphones, widely used for listening to PLDs, were examined. Three music genres-hip-hop ('Geogiseo geogi: Without you' by Dynamic Duo, 3 minutes and 59 seconds long), rock ('Sorry for party rocking' by LMFAO, 3 minutes and 24 seconds long), ballade ('Shigan-eul geosleo, Hae-reul peum-eun dal OST' by Lynn, 3 minutes and 31 seconds long) were played on the Samsung galaxy note MP3 player. These songs were selected because they were songs widely favored by high school students during the investigation.

The Bruel \& Kjaer Type 2250 Light with 1/2" microphone Type 4950 was used for output level and frequency analyses. Calibration of the equipment was performed by sound calibrator Type 4231 employing $1000 \mathrm{~Hz}$ at 94 and $114 \mathrm{~dB}$ sound pressure level (SPL) within $\pm 0.2 \mathrm{~dB}$ SPL error range. In order to formulate the ear canal space to resemble actual listening environments for accuarate measurments, 2 CC coupler Type 4946 was used for the ear-bud earphones and the Artificial Ear Type 41536 CC coupler was used for over-the-ear earphones (Fig. 1).

Output measurements were conducted in a quiet room. After linking earphones and appropriate couplers, the measurements were performed while the music was playing for at least 3 minutes long. The output level measurements were recorded in decibel A-weighted sound pressure level (dBA SPL). For frequency analysis, Z-weighted output levels (dB SPL) for all 1/3-octave frequency bands were recorded between 12.5 and $16000 \mathrm{~Hz}$. The center frequencies (CFs) of all 1/3-octave bands which produce the loudest were also recorded for the statistical analysis. The two volume settings of the Samsung galaxy note MP3 player were \#8 representing average volume around half $(53.3 \%)$ of the whole range and \#15 representing the maximum volume $(100 \%)$. All music were measured at both $\# 8$ and \#15 volume settings, and both maximum output level (LApk) 
and continuous equivalent output level (LAeq) were measured. While playing all music for at least 3 minutes, LApk was recorded when volume reached the maximum and LAeq was measured for overall average volume. Additionally, to search for safe volume levels, the closest volume level settings producing 65 and 85 dBA SPLs in LAeq depending on different earphone styles and music genres were examined. This range was selected because it represents safe and comfortable levels for both quiet and noisy environments according to previous literatures. $^{3,15)}$

All statistical analyses were performed using SPSS version 17.0 (SPSS Inc., Chicago, IL, USA) with alpha value less than 0.05 considered statistically significant. A $2 \times 3$ within-subjects repeated-measures ANOVA was used to analyze the output level data. There were two independent variables. One was earphone type which had 2 parameters; ear-bud and over-theear. The other was music gen-re which had 3 parameters; hiphop, rock, and ballade. The dependent variables were dBA SPLs measured in 'LApk' and 'LAeq' for both \#8 and \#15 volume settings. For analysis of CF, Friedman's ANOVA and Wilcoxon's signed rank test were used.

\section{Output Levels in dBA SPLs}

Twenty earphones' output levels according to the earphone types and music genres were measured and averaged at both maximum (\#15) and medium (\#8) volume settings (Table 1). Output levels in both LApk and LAeq were slightly higher for the ear-bud than the over-the-ear earphones at both volume settings. The repeated measures ANOVA revealed that the main effects of dBA SPLs were not significant in relation to the earphone type $[\mathrm{F}(1,18)=0.310 ; 0.012 ; 0.207 ; 0.022, p=$ $0.585 ; 0.913 ; 0.654 ; 0.885]$, but were significant in relation to the music genre $[\mathrm{F}(2,36)=8.002 ; 27.666 ; 4.118 ; 18.142, p=$ $0.000 ; 0.000 ; 0.025 ; 0.000]$ for all four dependent variables, LApk at maximum, LAeq at maximum, LApk at medium, and LAeq at medium volume settings. There was no significant interaction between earphone type and music genre for all dependent variables $[\mathrm{F}(2,36)=0.430 ; 0.096 ; 1.131 ; 1.055, p=0.654$; $0.909 ; 0.334 ; 0.359]$. The post-hoc analysis by the paired t-test was used to reveal the music genre contributing to the significant effects. For LAeq at both volume settings, significant differences between rock and other genres were found $[\mathrm{t}(19)=$ 8.950; 5.811; 4.919; 4.773, $p=0.000,0.000,0.000,0.000]$, but no significant differences between hip-hop and ballade $[\mathrm{t}(19)=$ $0.832 ; 0.920, p=0.416,0.369$ ] were found. For LApk at the maximum volume setting, significant differences were noted between hip-hop and other genres $[\mathrm{t}(19)=3.89 ; 4.155, p=0.001$, $0.001]$, but no significant difference between rock and ballade $[\mathrm{t}(19)=0.062, p=0.951]$ was revealed. For LApk at the medium volume setting, a significant difference between hip-hop and ballade was indicated $[\mathrm{t}(19)=2.972, p=0.008]$, but no significant differences between rock and other genres $[\mathrm{t}(19)=$ $1.377 ; 1.432, p=0.184 ; 0.168$ ] were revealed.

For each earphone type, output levels in dBA SPLs were compared according to the music genre. Output levels of LApk were the greatest in ballade music for both volume settings and both earphone types. Output levels of LAeq were the greatest
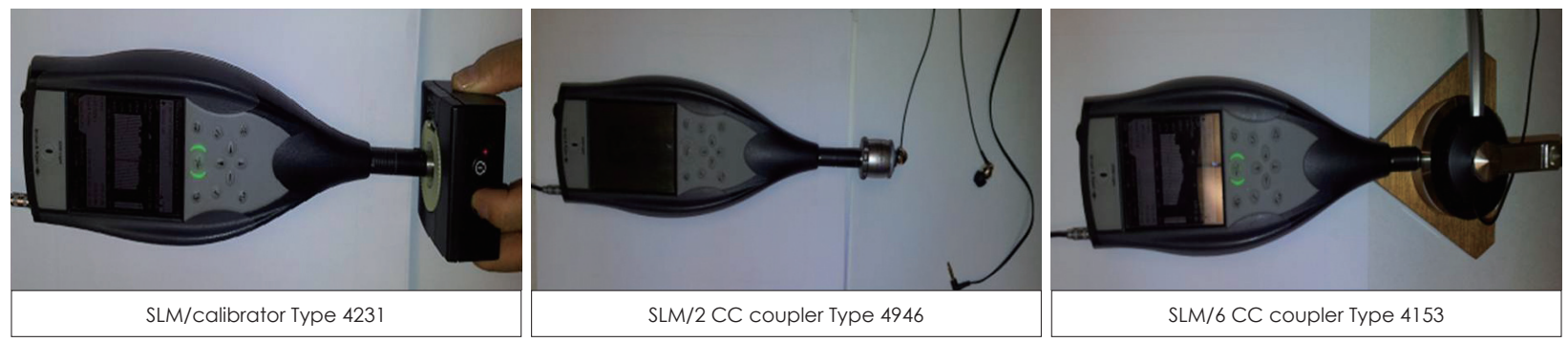

Fig. 1. Bruel \& Kjaer Type 2250 Light sound level meter and 2/6 CC coupler.

Table 1. Means and standard deviations of the output levels in ABA SPLs

\begin{tabular}{|c|c|c|c|c|c|c|c|c|c|}
\hline & \multirow{3}{*}{$\begin{array}{l}\text { Output } \\
\text { level }\end{array}$} & \multicolumn{8}{|c|}{ Earphone style } \\
\hline & & \multicolumn{4}{|c|}{ Over-the-ear } & \multicolumn{4}{|c|}{ Ear-bud } \\
\hline & & MR & $\mathrm{MH}$ & $M B$ & Total & $M R$ & $\mathrm{MH}$ & $M B$ & Total \\
\hline \multirow[t]{2}{*}{ Volume at maximum (\#15) } & LApk & $106.1(3.3)$ & $104.2(3.5)$ & $106.5(3.3)$ & $105.6(3.5)$ & $108.0(6.2)$ & $105.0(7.6)$ & $108.2(9.1)$ & $107.1(7.9)$ \\
\hline & LAeq & $101.8(3.4)$ & $98.9(3.7)$ & $99.1(3.2)$ & $99.9(3.7)$ & $102.0(7.8)$ & $99.1(7.5)$ & $99.7(8.9)$ & $100.3(8.2)$ \\
\hline \multirow[t]{2}{*}{ Volume at medium (\#8) } & LApk & $83.4(2.9)$ & $82.0(3.3)$ & $85.8(4.7)$ & $83.7(4.0)$ & $85.1(8.8)$ & $84.4(7.9)$ & $85.7(9.0)$ & $85.1(8.6)$ \\
\hline & LAeq & $79.7(3.1)$ & $76.7(3.5)$ & 77.7 (3.2) & $78.1(4.5)$ & $80.0(8.4)$ & $77.9(7.9)$ & $77.6(8.8)$ & $78.5(8.4)$ \\
\hline
\end{tabular}

Parentheses show the standard deviations. LAeq represents continuous equivalent SPL in dBA and LApk represents peak SPL in dBA. MR, MH, MB mean music genres of rock, hip-hop, ballade. dBA SPL: decibel A-weighted sound pressure level, LApk: maximum output level, LAeq: continuous equivalent output level 
Table 2. Volume level setting numbers out of full \#15 (100\%) and percentages for producing about 85 and 65 dBA SPLs in LAeq for each music genre

\begin{tabular}{lcrrrrrrr}
\hline & \multicolumn{4}{c}{ Over-the-ear } & \multicolumn{3}{c}{ Ear-bud } \\
\cline { 2 - 9 } & \multicolumn{1}{c}{ MR } & MH & MB & Total & MR & \multicolumn{1}{c}{ MH } & MB & Total \\
\hline Volume \# for 85 dBA SPL & $\# 9.8(65.3)$ & $\# 10.7(71.3)$ & $\# 10.2(68.0)$ & $\# 10.2(68.0)$ & $\# 9.5(63.3)$ & $\# 10.2(68.0)$ & $\# 10.2(68.0)$ & $\# 10.0(66.7)$ \\
Volume \# for 65 dBA SPL & $\# 3.4(22.7)$ & $\# 4.3(28.7)$ & $\# 4.1(27.3)$ & $\# 3.9(26.0)$ & $\# 3.8(25.3)$ & $\# 4.3(28.7)$ & $\# 4.2(28.0)$ & $\# 4.1(27.3)$ \\
\hline
\end{tabular}

LAeq represents equivalent level and MR, MH, MB mean music genres of rock, hip-hop, and ballade. Parentheses indicate the percentages (\%) of the full volume levels. dBA SPL: decibel A-weighted sound pressure level, LAeq: continuous equivalent output level

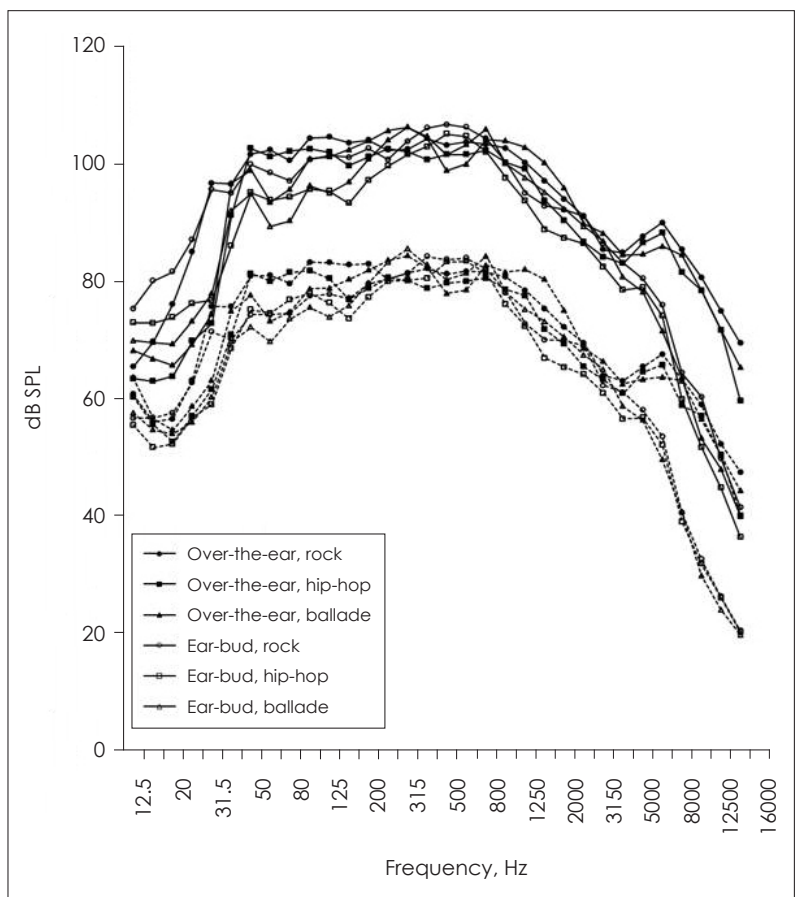

Fig. 2. Z-weighting output levels in $\mathrm{dB}$ SPLs as a function of 1/3-octave frequency bands at two volume settings, \#15 (solid line) and \#8 (dotted line), and two earphone styles, the ear-bud (open symbols) and over-the-ear (closed symbols) earphones for three music genres. SPL: sound pressure level.

in rock music for both volume settings and both earphone types.

For searching safe and comfortable volume settings, the closest volume level settings producing 65 and 85 dBA SPLs in LAeq were examined depending on different earphone types and music genres (Table 2). Volume levels for both 85 and 65 dBA SPLs were the lowest in rock music for both earphone types implying that the loudest music genre in average listening is rock music. However, the earphone types did not seem to affect the volume setting levels. For producing about 85 and 65 dBA SPLs, around \#10 (66.7\%) and \#4 (26.7\%) out of full \#15 (100\%) volume levels were recorded for both earphone types.

\section{Frequency Analysis at 1/3-Octave Bands}

At all 1/3-octave frequency bands, Z-weighting output lev-
Table 3. Means of center frequencies in $\mathrm{Hz}$ with respect to greatest dB SPLs of $1 / 3$ octave frequency band at two volume settings for two earphone styles and three music genres

\begin{tabular}{llllllll}
\hline & \multicolumn{3}{c}{ Over-the-ear } & & \multicolumn{3}{c}{ Ear-bud } \\
\cline { 2 - 3 } \cline { 6 - 7 } & MR & MH & MB & & MR & MH & MB \\
\hline Volume \#8 & 289 & 326 & 601 & & 516 & 560 & 604 \\
Volume \#15 & 330 & 322 & 429 & & 425 & 473 & 571 \\
\hline
\end{tabular}

$\mathrm{MR}, \mathrm{MH}, \mathrm{MB}$ mean music genres of rock, hip-hop, and ballade. SPL: sound pressure level

els in dB SPLs were displayed depending on volume settings, earphone styles, and music genres (Fig. 2). The maximum \#15 volume levels showed louder output levels than the medium \#8 volume levels at all frequency ranges as expected with similar configuration for both volume levels. On average, types of earphones did not show difference at 1/3-octave frequency bands except over $4000 \mathrm{~Hz}$. Over $4000 \mathrm{~Hz}$, output levels of the overthe-ear exceeded those of the ear-bud earphones with increasing difference toward $16000 \mathrm{~Hz}$. Regardless of the types of earphones and music genres, the output levels were substantial between 50 and $1000 \mathrm{~Hz}$ ranging about $80 \mathrm{~dB}$ SPL at the medium volume \#8 and $100 \mathrm{~dB}$ SPL for the maximum volume \#15.

The means of twenty earphones' CFs for $1 / 3$ octave bands were calculated with respect to the greatest $\mathrm{dB}$ SPLs at the medium \#8 and maximum \#15 volume settings. CFs were the highest in ballade music for both earphone types (Table 3). At the medium volume level, the CFs between the ear-bud and overthe-ear earphone types were not significantly different (Wilcoxon test, $\mathrm{Z}=1.244, p=0.214$ ). But the $\mathrm{CFs}$ among three music genres, rock, hip-hop, and ballade were significantly different [Friedman test, $\chi^{2}(2)=10.606, p=0.008$ ]. At the maximum volume level, however, the CFs were not significantly different between ear earphone styles (Wilcoxon test, $\mathrm{Z}=1.120$, $p=0.263$ ) and among the three music genres [Friedman test, $\chi^{2}$ $(2)=2.294, p=0.318]$.

\section{Conclusion}

The results found that the earphone styles did not affect output levels but music genres did. Further, the post-hoc analysis revealed that rock music, which was discovered to be the loudest, showed difference at LAeq whereas hip-hop music, which was discovered to be the softest, showed the difference at LApk. 
A comparison of the mean values showed the ballade music had the greatest output level at LApk. For both earphone styles, output levels at about 85 and $65 \mathrm{dBA}$ SPLs which are assumed to be safe were around $\# 10(66.7 \%)$ and $\# 4(26.7 \%)$ out of full \#15 (100\%) volume level setting.

At all 1/3-octave frequency bands between 12.5 and 16000 $\mathrm{Hz}, \mathrm{dB}$ SPLs were substantial between 50 and $1000 \mathrm{~Hz}$ regardless of the styles of earphones and music genres. The types of earphones did not show difference except when over $4000 \mathrm{~Hz}$. Over $4000 \mathrm{~Hz}$, output levels of the over-the-ear exceeded those of the ear-bud earphones. The CFs producing the loudest output level recorded the highest frequency in ballade music for both earphone styles. At the maximum volume level, $\mathrm{CFs}$ were not affected by earphone styles or music genres. However, at the medium volume level, earphone types did not affect CFs but music genres did.

The output levels of the earphone types were not different in this investigation, although many researchers reported that the ear-bud earphones could increase $5-9 \mathrm{dBs}$ in output levels comparing to over-the-ear earphones. ${ }^{4,13,14)}$ The reason for this discrepancy can be found in the method of measurements. While this investigation used couplers considering 3 dimensional construct of ears, advanced studies simulated actual listening appearance using Knowels Electronic Manikin for Acoustical Research and a head and torso simulator. As microphone got nearer to earphone for actual listening appearances, which describes intensity of the output levels may have increased obeying an inverse-square law, how a specified physical intensity is inversely proportional to square of the distance from the source of that physical quantity. Therefore, this investigation concludes that ear-bud earphones themselves are not responsible for louder output levels. It can be implied that the increased output levels of ear-bud earphones are due to distance and passive sound isolation when we listen to music. Thus, isolation by covering of ears by over-the-ear earphone can lower the volume in noisy background. ${ }^{11)}$

Contrary to recommended earphone type, over-the-ear earphones for protecting hearing ability, ${ }^{4)}$ the majority of cell phones, PLDs, and MP3 players provide ear-bud earphones in the market. Therefore, consumers are more exposed to the danger of MIHL and even presbycusis in the case of young adults. ${ }^{9)}$ There are two ways to be safe while using ear-bud earphones: lowering volume and extending distance to tympanic membrane. Sound isolation must be assured to lower volume levels without sacrificing quality of music in background noise while extending distance to tympanic membrane.

The effects of music genres for output levels were found to be significant in this investigation. However, inconsistency of findings in this field is noted. While no significant difference among samples of rock, $R \& B$, country, dance, and top forty music genres were reported at maximum volume settings, ${ }^{13)}$ rock, country, and adult contemporary music samples showed louder levels than samples of rap/R\&B, pop, classical, dance, and jazz. ${ }^{4)}$ The latter finding was supported by the results of this investigation in which rock was the loudest music genre at equivalent measuring. Surprisingly, in this investigation, ballade music which was deemed relatively quiet was the loudest at its peak. In conclusion, the music genre does not seem to affect the output levels of PLDs considerably except hard rock. In fact, considering the frequency spectrum was affected remarkably according to music genre for instrument used, the output levels of music may lie on combinations of the music instruments used and their peak levels.

Most people listen to the music at about $50-60 \%$ maximum volume setting. ${ }^{7)}$ Accordingly, out of 15 volume range, $50-60 \%$ volume setting ( $\# 8$ volume) was investigated in this study. The peak sound level output was 83.7 and $85.1 \mathrm{dBA}$ SPLs and equivalent sound level output was 78.1 and $78.5 \mathrm{dBA}$ SPLs for over-the-ear and ear-bud earphones, respectively. These levels are not considered safe. Occasionally, the sound level reached over 85 dBA SPLs at \#8 volume settings during the experiment. To produce about 85 and $65 \mathrm{dBA}$ SPL for both earphone types, around \#10 (66.7\%) and \#4 (26.7\%) volume levels were measured, respectively. With these result, it can be stated that the volume setting of PLDs should be far lower than $50 \%$ of maximum volume in daily listening for our hearing health. When listening for long periods of time, volume levels should be around \# 4 (26.7\%).

During the experiment, the need for more information about a product by manufacturers was noted. Other than manufacturers and model name, volume and frequency ranges of most MP3 players should be labeled on earphones on little attached flap or hard tap. Manufacturers also can upload smart cell phone accessory earphones' information on applications. It is strongly recommended that the MP3 player statistically presents accumulated listening time and sound levels on its screen. Making software that warns listeners when they listen to over 85 dBA SPLs for long periods of time is also recommended. This way, the consumers can know about the degree of sound levels produced by earphones ability and be cautious about damaging their ears.

To refine conclusions in the future, more earphones and diverse songs in various music genres should be analyzed because this investigation included only limited numbers of earphones and songs for experiments. The effect of frequency spectrums to the hearing system after music listening should also be given additional research. Analyzing the effect of the mixture of output level and frequency spectrum to the hearing sys- 
tem will also help improve our understanding of relation between sound from PLDs and our hearing health.

\section{REFERENCES}

1) Clark WW. Hearing: the effects of noise. Otolaryngol Head Neck Surg 1992;106:669-76.

2) National Institute of Health. Noise and Hearing loss. NIH Consensus Development Conference Consensus Statement. Bethesda, MD: NIH; 1990. p.22-4.

3) Airo E, Pekkarinen J, Olkinuora P. Listening to music with earphones: an assessment of noise exposure. Acta Acoust 1996;82:885-94.

4) Fligor BJ, Cox LC. Output levels of commercially available portable compact disc players and the potential risk to hearing. Ear Hear 2004; 25:513-27.

5) Portnuff CD, Fligor BJ, Arehart KH. Teenage use of portable listening devices: a hazard to hearing? J Am Acad Audiol 2011;22:663-77.

6) Williams W. Noise exposure levels from personal stereo use. Int J Audiol 2005;44:231-6

7) Keith SE, Michaud DS, Chiu V. Evaluating the maximum playback sound levels from portable digital audio players. J Acoust Soc Am 2008;123:4227-37.

8) Kumar A, Mathew K, Alexander SA, Kiran C. Output sound pressure levels of personal music systems and their effect on hearing. Noise Health 2009;11:132-40.
9) Kujawa SG, Liberman MC. Acceleration of age-related hearing loss by early noise exposure: evidence of a misspent youth. J Neurosci 2006;26:2115-23.

10) Zogby International. Survey of teens and adults about the use of personal electronic devices and head phones. http://www.asha.org/NR/ rdonlyers/10B67FA1-002C-4C7B-BAOB-ICOA3AF98A63/O/zogbysurvey2006.pdf. WWW Document;2006.

11) Hodgetts WE, Rieger JM, Szarko RA. The effects of listening environment and earphone style on preferred listening levels of normal hearing adults using an MP3 player. Ear Hear 2007;28:290-7.

12) Muchnik C, Amir N, Shabtai E, Kaplan-Neeman R. Preferred listening levels of personal listening devices in young teenagers: self reports and physical measurements. Int J Audiol 2012;51:287-93.

13) Portnuff CDF, Fligor BJ. Sound output levels of the iPod and other MP3 players: Is there potential risk to hearing? Paper presented at the Noise-Induced Hearing Loss in Children meeting, Cincinnati, OH. [FTP archive] 2006 October. Available from: URL: http://hearingconservation.org/displaycommon.

14) Keppler H, Dhooge I, Maes L, D'haenens W, Bockstael A, Philips B, et al. Short-term auditory effects of listening to an MP3 player. Arch Otolaryngol Head Neck Surg 2010;136:538-48.

15) Fligor BJ, Ives TE. Does headphone type affect risk for recreational noise-induced hearing loss? Paper presented at the Noise-Induced Hearing Loss in Children meeting, Cincinnati, OH. [FTP archive] 2006, October. Available from: URL: http://hearingconservation.org/ displaycommon. 